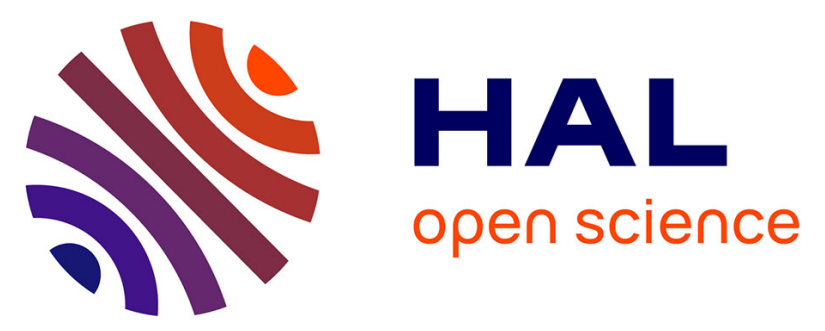

\title{
Synthesis and structure of free-standing germanium quantum dots and their application in live cell imaging
} Ali Karatutlu, Mingying Song, Ann P. Wheeler, Osman Ersoy, William R. Little, Yuanpeng Zhang, Pascal Puech, Filippo S. Boi, Zofia Luklinska, Andrei V. Sapelkin

\section{To cite this version:}

Ali Karatutlu, Mingying Song, Ann P. Wheeler, Osman Ersoy, William R. Little, et al.. Synthesis and structure of free-standing germanium quantum dots and their application in live cell imaging. RSC Advances, 2015, 5 (26), pp.20566-20573. 10.1039/c5ra01529d . hal-01756982

\section{HAL Id: hal-01756982 https://hal.science/hal-01756982}

Submitted on 3 Apr 2018

HAL is a multi-disciplinary open access archive for the deposit and dissemination of scientific research documents, whether they are published or not. The documents may come from teaching and research institutions in France or abroad, or from public or private research centers.
L'archive ouverte pluridisciplinaire $\mathbf{H A L}$, est destinée au dépôt et à la diffusion de documents scientifiques de niveau recherche, publiés ou non, émanant des établissements d'enseignement et de recherche français ou étrangers, des laboratoires publics ou privés. 


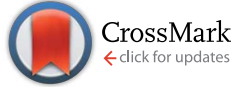

Cite this: RSC Adv., 2015, 5, 20566

Received 26th January 2015

Accepted 13th February 2015

DOI: $10.1039 / \mathrm{c} 5 \mathrm{ra01529d}$

www.rsc.org/advances

\section{Synthesis and structure of free-standing germanium quantum dots and their application in live cell imaging $\dagger$}

\author{
Ali Karatutlu, ${ }^{\text {*af }}$ Mingying Song, ${ }^{a}$ Ann P. Wheeler, ${ }^{b}$ Osman Ersoy, ${ }^{a}$ William R. Little, ${ }^{a}$ \\ Yuanpeng Zhang, ${ }^{a}$ Pascal Puech, ${ }^{c}$ Filippo S. Boi, ${ }^{d}$ Zofia Luklinska ${ }^{e}$ \\ and Andrei V. Sapelkin ${ }^{a}$
}

Free-standing Ge quantum dots around $3 \mathrm{~nm}$ in size were synthesized using a bench-top colloidal method and suspended in water and ethanol. In the ethanol solution, the photoluminescence of the Ge quantum dots was observed between 650 and $800 \mathrm{~nm}$. Structural and optical properties of these colloidal Ge quantum dots were investigated by utilizing X-ray diffraction, X-ray absorption spectroscopy, Raman spectroscopy, and photoluminescence spectroscopy and transmission electron microscopy. The structure of the as-prepared Ge quantum dots that were found is best described by a core-shell model with a small crystalline core and an amorphous outer shell with a surface that was terminated by hydrogen-related species. As-prepared Ge quantum dots were suspended in cell growth medium, and then loaded into cervical carcinoma (HeLa) cells. The fluorescent microscopy images were then collected using $405 \mathrm{~nm}, 488 \mathrm{~nm}, 561 \mathrm{~nm}$ and $647 \mathrm{~nm}$ wavelengths. We observed that, based on fluorescence measurements, as-prepared Ge quantum dots can remain stable for up to 4 weeks in water. Investigation of toxicity, based on a viability test, of as-prepared uncoated Ge quantum dots in HeLa cells was carried out and compared with the commercial carboxyl coated CdSe/ZnSe quantum dots. The viability tests show that Ge quantum dots are less toxic when compared to commercial carboxyl coated CdSe/ZnS quantum dots.

\section{Introduction}

Real time monitoring of a cell's function and state can be carried out by using visible and near-infrared emitting luminescent nano-scale particles, which are also known as quantum dots (qdots). ${ }^{\mathbf{1}, 2}$ The repetition and reliability of the luminescence at room temperature from various inorganic qdots make them excellent candidates for the detection of

${ }^{a}$ Centre, for Condensed Matter and Materials Physics, School of Physics and Astronomy, Queen Mary, University of London, London, E1 4NS, UK. E-mail: a. karatutlu@qmul.ac.uk

${ }^{b}$ Blizard Institute of Cell and Molecular Sciences, Barts and the Royal London Hospital School of Medicine and Dentistry, Queen Mary, University of London, E1 2AT, UK

${ }^{c}$ CEMES-CNRS, University of Toulouse, 29 rue Jeanne Marvig, 31055 Toulouse, France ${ }^{d}$ School of Engineering and Materials Science, Queen Mary, University of London, London, E1 4NS, UK

${ }^{e}$ School of Physical Science and Technology, Sichuan University, 29 Wangjiang road, 610064, People's Republic of China

${ }^{f}$ Electrical and Electronics Engineering, Bursa Orhangazi University, YILDIRIM Campus, 16310, Yildirim, Bursa, Turkey.E-mail: ali.karatutlu@bou.edu.tr

$\dagger$ Electronic supplementary information (ESI) available: Including table of laser power of reflection and incident light for the fluorescence images taken using spinning disk confocal microscopy, the detailed procedure and structural parameters of the EXAFS fit, Raman spectroscopy results and table of size analyses by Raman, TEM and XRD. See DOI: 10.1039/c5ra01529d biomolecular interplay and imaging applications in vivo. ${ }^{2-6}$ qdots also have a high resistivity to photobleaching and their narrow emission and broad absorption bands can be tuned by varying the particle size and composition. Thus, quantum yield, small size and lack of photobleaching are some of the reasons why qdots are becoming a popular alternative to fluorescent organic dyes for biological fluorescent imaging., CdSe/ZnS-based qdots currently hold the dominant position in bio-imaging applications. However, $\mathrm{CdX}(\mathrm{X}=\mathrm{Se}, \mathrm{Te})$ qdots show significant levels of cytotoxicity when used in cell imaging/diagnostics. ${ }^{8,9}$ When CdSe has been coated with ZnS there is still a considerable cytotoxic effect. ${ }^{10}$ At the end of $24 \mathrm{~h}$, approximately $40 \%$ of the macrophage cells was observed as killed even with small concentrations of CdSe/ZnS qdots (e.g. $2.5 \mathrm{nM}$ ).

An alternative system is the InP qdots, however, production of high quality qdots is more challenging for these materials than for the Cd-based systems. ${ }^{3}$ Group 4 materials (C, Si and Ge) can offer a viable alternative due to relatively low toxicity. ${ }^{\mathbf{6}, 11,12}$ Their optical properties can also be radically enhanced by exploiting the quantum confinement effect (QCE). ${ }^{\mathbf{1 3 - 1 6}}$ Ge qdots are expected to show these unique properties in accordance with the QCE for relatively large particles due to the fact that its exciton Bohr radius is estimated to be larger $\left(R_{\mathrm{B}}=24.3 \mathrm{~nm}\right)$ 
than that of $\mathrm{Si}\left(R_{\mathrm{B}}=4.9 \mathrm{~nm}\right) \cdot{ }^{17}$ One of the fundamental problems that preclude the wider use of $\mathrm{Si}$ and $\mathrm{Ge}$ in imaging application is an indirect band gap that results in a relatively inefficient light emission. However, this can be improved by the QCE as has originally been demonstrated for porous Si by L. Canham in 1991. ${ }^{13,14}$ Modifying the surface species can also alter the optical properties of these qdots and can improve their stability by saturating the dangling bonds for instance by hydrogen or carbon atoms. ${ }^{18,19}$ For over 20 years various physical and chemical routes of the synthesis of Ge qdots have been reported. ${ }^{15,20,21,17,22-29} \mathrm{~A}$ novel and significant method of colloidal synthesis, particularly reducing the halides $\left(\mathrm{GeI}_{2} / \mathrm{GeI}_{4}\right)$, was recently reported as the most elegant method in terms of size/shape modification and the stability of Ge qdots. ${ }^{28-30}$ However, this method requires the high temperature Schlenk technique which makes it rather elaborate. Recently, colloidal synthesis methods ${ }^{26,30}$ have become available using room temperature benchtop chemistry. These methods seem to yield small Ge qdots and allow for some control over size selectivity as well. However, potential suitability of these Ge qdots for bio-applications would significantly depend on their atomic structure, morphology and surface termination as each of these physiochemical properties are essential to understand the toxicity of qdots. ${ }^{31}$ For example, oxygen-terminated Ge qdots may have limited use in bio-application due to water solubility of germanium oxide. It has been suggested ${ }^{26}$ that, following the benchtop colloidal synthesis, the structure of as-prepared Ge qdots is amorphous (based on the X-ray diffraction), while the sample seems to be crystalline according to the selective area electron diffraction (SAED) results. ${ }^{27}$ The latter may suggest a transformation to a crystalline phase due to annealing in the highly energetic electron beams used in TEM. The nature and stability of the surface is also unclear. Furthermore, there are some reports suggesting a Ge tetragonal phase (ST-12 phase) may be obtained upon deposition by the cluster beam evaporation technique,$^{32,33}$ releasing of high pressure ${ }^{34}$ or annealing at high temperature. There are also some studies including lithiation $^{35}$ and aging ${ }^{36}$ that show ST-12 phases mixed together either with lithium atoms or the diamond cubic phase of Ge. The ST-12 phase has a potential for optoelectronic applications since it is predicted to be a direct band gap material $\left(E_{\mathrm{g}}=1.47 \mathrm{eV}\right){ }^{37}$

Despite the potential in the biomedical applications of $\mathrm{Ge}$ qdots there are few studies on biological imaging and the toxicity of Ge qdots. ${ }^{38,39}$ Herein we present a slightly modified method of the preparation of Ge qdots to yield colloidally stable Ge qdots. We use a combination of direct visual techniques, such as TEM, together with short range (X-ray absorption spectroscopy, Raman and long range (XRD, Raman) sensitive structural methods to investigate atomic arrangements on the sub-nanoscale. We test biocompatibility of Ge qdots using the viability test and compare it with the commercial carboxyl coated CdSe/ZnS qdots. We also assess the potential of Ge qdots for cell imaging applications.

\section{Experimental}

\section{Materials and methods}

Materials. The chemicals, $\mathrm{GeCl}_{4}(>99 \%)$, ethylene glycol (ETG, $99 \%$ ), and $2 \mathrm{M}$ of sodium borohydride solution in triethylene glycol dimethyl ether ('the triglyme') were used as purchased from Sigma-Aldrich. Polyvinylpyrolidine (PVP) (MW $=630.000)$ were used as purchased from Tokyo-chemicals.

\section{Synthesis of Ge qdots}

Ge qdots, named as $\mathrm{CS}_{1}$, were synthesized by a slight modification of a benchtop colloidal synthesis route ${ }^{27}$ which produces $\mathrm{CS}_{1}$ suspended in water and ethanol. In the first part of the synthesis $265 \mu \mathrm{L}$ of $\mathrm{GeCl}_{4}$ were reduced using a solution of $10 \mathrm{ml}$ of ETG and $50 \mathrm{mg}$ of PVP. $6 \mathrm{ml}$ of the triglyme was then added at a rate of $90 \mathrm{ml} \mathrm{h}^{-1}$ for the first $2 \mathrm{ml}$ and then $9 \mathrm{ml} \mathrm{h}^{-1}$ for the remaining $4 \mathrm{ml}$. This controlled addition process of the triglyme was performed with a syringe pump into a 3 neck round bottom beaker in which the solution was bubbled using a continuous $\mathrm{H}_{2} \mathrm{Ar}(5 \% / 95 \%)$ gas flow with an inlet of a micro-tube through the solution. The formation process took approximately $1 \mathrm{~h}$ and the final product was separated from the colloidal chemical solution by $10 \mathrm{~min}$ of centrifugation at $10000 \mathrm{rpm}$.

\section{Characterization of Ge qdots}

After the synthesis, Raman spectroscopy and photoluminescence (PL) spectroscopy measurements were conducted with a Renishaw 1000 spectrometer. A diode laser at a wavelength of $473 \mathrm{~nm}$ was used for $\mathrm{CS}_{1}$, whereas for the $\mathrm{Ge}-\mathrm{H}$ stretching mode of $\mathrm{CS}_{1}$, a He-Ne laser at a wavelength of $633 \mathrm{~nm}$ was used. TEM measurements were taken using a JEOL 2010 to characterize the morphology and the size of $\mathrm{CS}_{1}$. Gatan digital micrograph software was used for the size analysis by TEM. XRD and EXAFS measurements were conducted in the beamline B18 (ref. 40) at Diamond Light Source in the UK. The EXAFS measurement was carried out at $T=100 \mathrm{~K}$ using a cryojet system. ATHENA ${ }^{41}$ was used to extract the absorption spectrum from the raw data. Then, the structural parameters were determined using the least squared fit of the EXAFS data in $r$ space with ARTEMIS ${ }^{41}$ by FEFF6 code $^{42}$ within the fitting range of the photoelectron momentum $(k)$ and the non-phase corrected radial distribution distance $(r)$ of $3-16 \AA^{-1}$ and 1.67-2.55 Å respectively. Energy resolution of EXAFS experiment was $1 \mathrm{eV}$.

\section{Cell culture}

The cervical carcinoma cells (HeLa cells) were cultured in a growth medium (89\% high glucose DMEM, 10\% Foetal Calf Serum, $1 \%$ Penicillin \& Streptomycin) with various densities inside 6-well plates according to the following trials. Cells were split once a week and incubated in a $5 \% \mathrm{CO}_{2}$ atmosphere at $37^{\circ} \mathrm{C}$. In the fixation steps, cells were firstly washed 2 times with phosphate buffered saline solution (PBS), and incubated with $2 \mathrm{ml}$ of $4 \%$ paraformaldehyde solution (PFA) per well for $10 \mathrm{~min}$ at room temperature, followed by washing 3 times with PBS. 
Fixed cells dishes or plates were then stored in a fridge for $24 \mathrm{~h}$ before post procedures.

\section{Visualization of Ge qdots in HeLa cells}

In order to investigate the Ge qdots impacts on HeLa cells, sample of HeLa cells with Ge qdots were prepared and imaged. HeLa cells were seeded on a glass coverslip in a 6-well plate at a density of $5.0 \times 10^{3}$ cells per $\mathrm{ml}$ per well, incubated overnight with 50 nmole of Ge qdots per well. Ge qdots were synthesized, suspended in water and sterilized under UV light for $15 \mathrm{~min}$. Ge qdots were re-suspended in growth medium. Ge qdots with different concentrations suspended in growth medium were loaded to the HeLa cells. The cells were fixed with PFA prior to fluorescence imaging.

Fluorescence images were acquired on a spinning disk confocal microscopy (consisting of a Nikon ECLIPSE TE2000-s microscope, a YOKOGAWA CSU-x1 spinning disk, and a $100 \times$ objective, NA 1.4). Sample was excited with four different wavelength lasers (405 nm, $488 \mathrm{~nm}, 561 \mathrm{~nm}$ and $640 \mathrm{~nm}$ ). Images were captured using an Andor-iXon3 885 camera and data were post processed on ImageJ.

The incoming laser was modulated through an acoustooptical tunable filter (AOTF) before being recorded by CCD camera. The reflected light from a mirror sample slide were recorded using an optical power meter (Newport 1916 C).

\section{Cell viability test}

Once the optical stability of Ge qdots $\left(\mathrm{CS}_{1}\right)$ in cells was confirmed the toxicity test was performed. Series of viability test were taken and each test were repeated 3 times.

To examine the toxicity, fresh $\mathrm{CS}_{1}$ samples were produced and tested on HeLa cells. HeLa cells were cultivated as described in section 2.3. Cells were seeded into a 12-well plate at a density of $5.0 \times 10^{3}$ cells per ml per well. Ge qdots were added into different wells after a whole night, at the concentration of 0 , 10, 100, 200, 300, 400, 500, 600 nmole. Viability of HeLa cells were tested on a MuseTM Analyser after $24 \mathrm{~h}$. In every viability measurement, at least 1000 cells were counted.

Regarding the long term effect of Ge qdots on live cells viability, HeLa cells were cultivated on a 12-well plate at a density of $15.0 \times 10^{3}$ cells per ml per well. $\mathrm{CS}_{1}$ was diluted into two concentration solutions of 25 nmole, 250 nmole.

Cell viability was analysed at 24,48 and $72 \mathrm{~h}$. Simultaneously, comparison tests were performed with commercial qdots (Qdot 625 ITK Invitrogen A10200:CdSe/ZnS core and shell structure, emission peak at $625 \mathrm{~nm})$. In every viability measurement, at least 1000 cells were counted.

\section{Results and discussion}

Scheme 1 demonstrates how the experiment was conducted and a brief formation mechanism of Ge qdots $\left(\mathrm{CS}_{1}\right)$ reduced from $\mathrm{GeCl}_{4}$. $\mathrm{CS}_{1}$ were prepared by nucleation of $\mathrm{Ge}$ atoms liberated by the reduction of $\mathrm{GeCl}_{4}$ (ref. 27) with a slight modification as designated in the scheme.

A possible formation mechanism of Ge qdots reducing from $\mathrm{GeCl}_{4}$ is outlined as follows:

$$
\begin{gathered}
\mathrm{BH}_{4}^{-}+\mathrm{GeCl}_{4} \rightarrow \mathrm{GeCl}_{4} \mathrm{H}^{-}+\mathrm{BH}_{3} \\
2\left(\mathrm{GeCl}_{4} \mathrm{H}^{-}\right) \rightarrow 2 \mathrm{Cl}^{-}+2\left(\mathrm{HGeCl}_{3}\right) \\
2\left(\mathrm{HGeCl}_{3}\right) \rightarrow 2\left(\mathrm{GeCl}_{2}\right)+2 \mathrm{HCl} \\
\mathrm{H}_{x} \mathrm{Cl}_{y}+\mathrm{GeCl}_{2} \rightarrow \mathrm{GeH}_{x} \mathrm{Cl}_{y}
\end{gathered}
$$

In the process of the production of Ge qdots by the colloidal synthesis route, the reaction is initiated upon a hydrophilic attack of $\mathrm{BH}_{4}{ }^{-}$to $\mathrm{GeCl}_{4}$ as a source of a hydride ion (eqn (1)) which can then lead to the formation of $\mathrm{GeCl}_{3} \mathrm{H}$ (eqn (2)). Then, the removal of $\mathrm{HCl}$ from $\mathrm{GeCl}_{3} \mathrm{H}$ (eqn (3)) could form reactive germanium(II) species such as $\mathrm{GeCl}_{2}$, which could then be

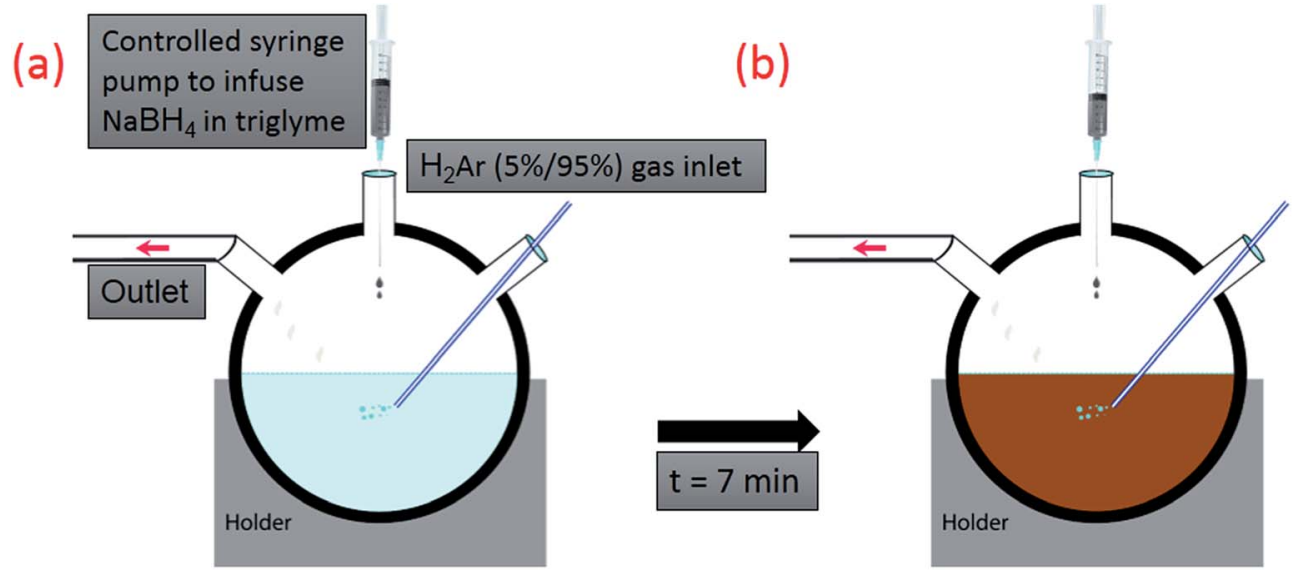

Scheme 1 Schematic illustration of formation of luminescent Ge qdots by colloidal synthesis. (a) $\mathrm{GeCl}_{4}, \mathrm{PVP}$ and ethylene glycol mixed before infusing $\mathrm{NaBH}_{4}$ in the triglyme into the solution (see experimental procedure for the details) Here, instead of $\mathrm{Ar}$ gas $26, \mathrm{H}_{2} \mathrm{Ar}(5 \% / 95 \%)$ gas were used to purge the solution in order to prevent possible oxide formation (b) $2 \mathrm{ml}_{\text {of }} \mathrm{NaBH}_{4}$ in the triglyme was added to the solution initially with the rate of $90 \mathrm{ml}$ per min using a syringe pump. Then, the rate was reduced to $9 \mathrm{ml} \mathrm{min}{ }^{-1}$ for the rest $4 \mathrm{ml}^{\text {of }} \mathrm{NaBH}_{4}$ in the triglyme. 
inserted into $\mathrm{GeH}_{x} \mathrm{Cl}_{y}$ molecular clusters (eqn (4)) to act as intermediates in the formation of Ge nanoparticles.

The images of $\mathrm{CS}_{1}$ immediately after production, and suspended in ethanol, are shown in Fig. 1(a). $\mathrm{CS}_{1}$, which was then placed onto a quartz boat after being dried by Ar gas, can also be seen in Fig. 1(b). The particles are yellow in color.

Raman spectroscopy was used as a first analysis technique to understand the structural properties of $\mathrm{CS}_{1}$ as well as to estimate the average size of particles. Raman spectroscopy measurements are shown together with the analysis of the data in Fig. 1(d). We observed a broad asymmetric peak just below $300 \mathrm{~cm}^{-1}$ as expected for diamond-type Ge qdots. ${ }^{20}$ In addition to the shift in the peak position relative to the bulk Ge optical phonon mode $\left(300 \mathrm{~cm}^{-1}\right),{ }^{43}$ the Raman spectrum of freestanding $\mathrm{CS}_{1}$ (see Fig. 1(d)) has an asymmetrical Lorentzianlike shape which is an indication that the sample is nanocrystalline. $^{23,44}$ We used the well-known Richter-FauchetCampbell (RFC) model ${ }^{45,46}$ based on phonon confinement to estimate the mean size of $\mathrm{CS}_{1}$ by fitting the corresponding phonon confinement expression to the data. We obtained the particle size to be approximately $3.2 \mathrm{~nm}$ for the Raman spectrum given in Fig. 1(d). The residual signal between the RFC model and the experimental spectrum is also shown in Fig. 1(d). There is a broad peak close to $250 \mathrm{~cm}^{-1}$. Possible origins of this residual might be due to (i) limitation of the phonon confinement model for very small nanoparticles, ${ }^{47}$ (ii) amorphous component $^{48}$ or (iii) a metastable phase..$^{36,49}$

The inset in Fig. 1(d) shows a broad peak centered at about $2000 \mathrm{~cm}^{-1}$ that was assigned to the Ge-H stretch mode of $\mathrm{CS}_{1}$. Hydride termination of Ge nanocrsytals using FT-IR studies was reported by several authors with a broad stretching mode between $1900 \mathrm{~cm}^{-1}$ to $2100 \mathrm{~cm}^{-1} .^{\mathbf{5 0 , 5 1 , 1 8 , 5 2}}$ As a complementary technique, EDS data was utilised and shows the traces of Ge (see Fig. S5 in ESI $\dagger$ ).

In Fig. 2(a), the TEM results are shown for $\mathrm{CS}_{1}$. The TEM micrograph of $\mathrm{CS}_{1}$ in Fig. 2(a) shows the mean size of $\mathrm{CS}_{1}$ to be $3.68 \mathrm{~nm} \pm 0.62 \mathrm{~nm}$ with a very narrow size distribution from out of 200 qdots of $\mathrm{CS}_{1}$ in Fig. 2(c). Comparison of the size analysis
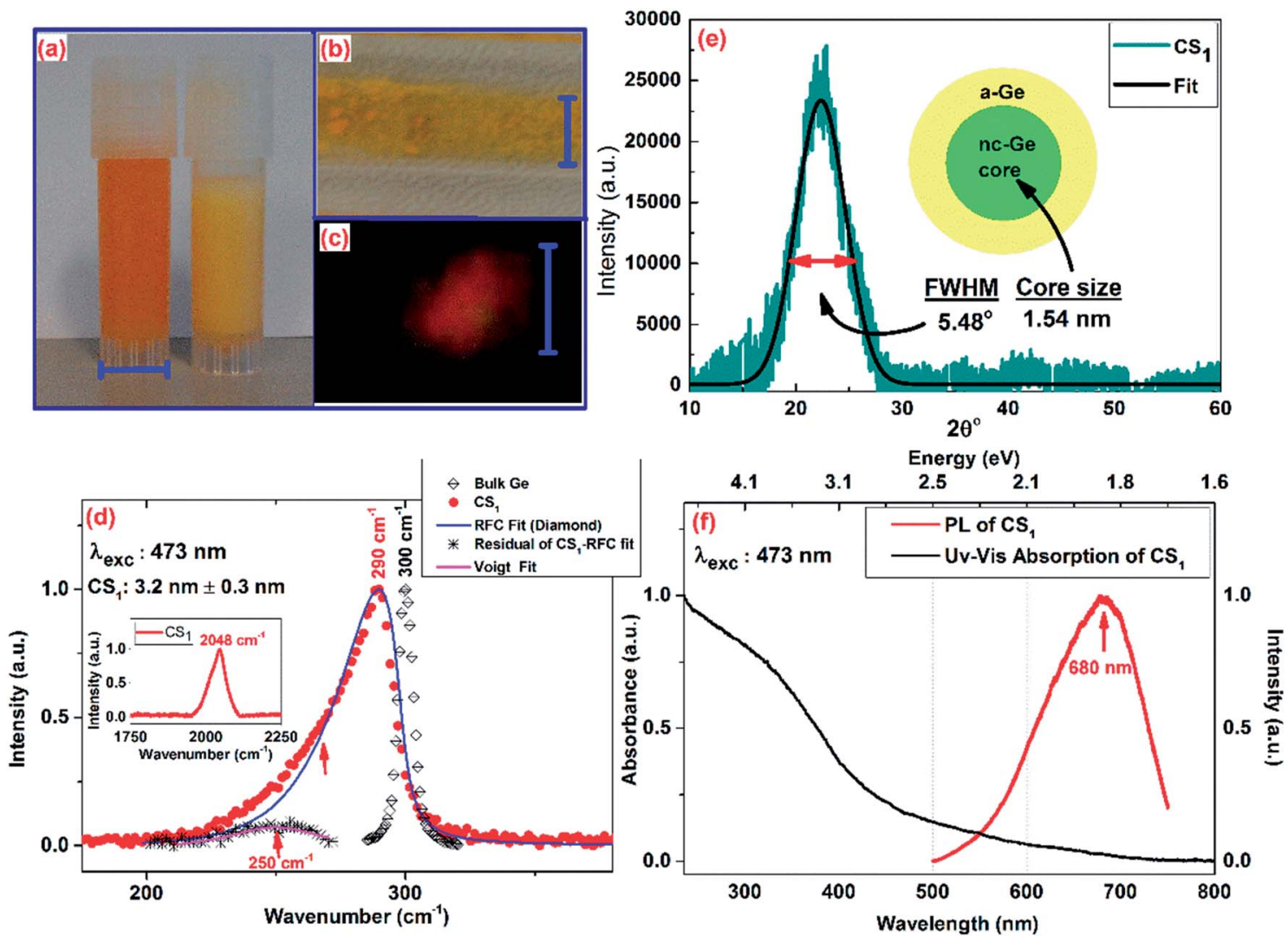

Fig. 1 (a) Picture of Ge qdots $\left(\mathrm{CS}_{1}\right)$ as-prepared and suspended in ethanol after centrifugation (b) Ge qdots in powder form after dried with Ar gas (c) luminescent picture of red emitting Ge qdots (the excitation wavelength was $325 \mathrm{~nm}$ ). Scale bars (all with blue colors) in (a), (b) and (c) are $10 \mathrm{~mm}, 17 \mathrm{~mm}$ and $0.75 \mathrm{~mm}$ respectively. (d) Raman shift of $\mathrm{CS}_{1}$ (red circles) shows an asymmetric Lorentzian peak positioned at $290 \mathrm{~cm}^{-1}$ obtained after the RFC fit (blue color). Residual between Raman data of $\mathrm{CS}_{1}$ and the RFC fit is shown and fitted with a Voigt fit (magenta color) which results in peak position of $250 \mathrm{~cm}^{-1}$. Raman of bulk Ge (black colored diamond) is shown to have a peak position at $300 \mathrm{~cm}^{-1}$. In a-Ge, there is one but a very broad and asymmetric peak ${ }^{65}$ at $275 \mathrm{~cm}^{-1}$. (See ESI $\uparrow$ for Raman of a-Ge reproduced from ref. 65). The inset in (d) shows stretching mode at $2048 \mathrm{~cm}^{-1}$ between Ge and $\mathrm{H}$. For Raman shift and photoluminescent spectroscopy measurements, an excitation wavelength of $473 \mathrm{~nm}$ was utilized. (e) X-ray diffraction (XRD) ( $\lambda=1.544 \AA, E=8047 \mathrm{eV}$ ) (f) normalized UV-Vis absorption spectroscopy and photoluminescence spectroscopy measurements. The inset core-shell schematic in (e) clarifies the discrepancy between Raman and XRD size calculations such as $3.2 \mathrm{~nm}$ and $1.54 \mathrm{~nm}$ respectively. 

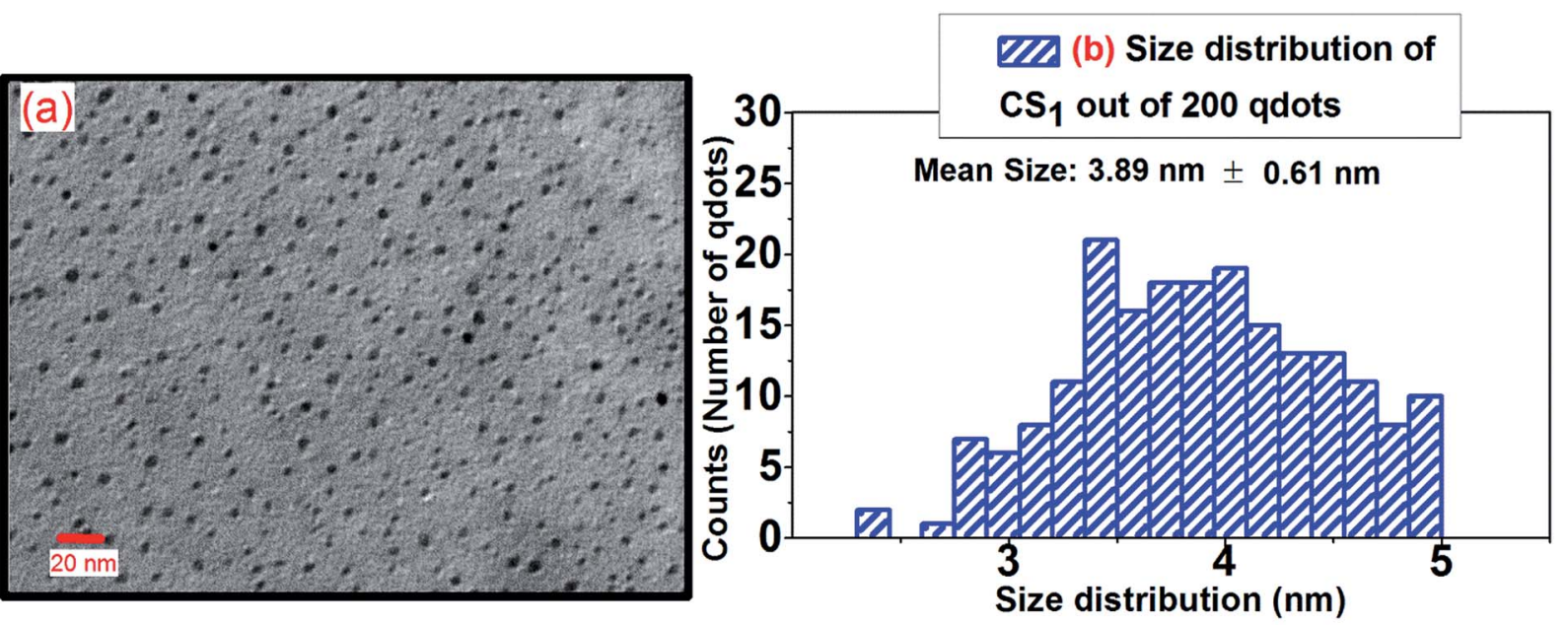

Fig. 2 (a) TEM micrograph of $\mathrm{CS}_{1}$. The graph in (b) is the size distribution of Ge qdots out of 200 qdots. The mean size of Ge qdots was found to be $3.68 \mathrm{~nm} \pm 0.62 \mathrm{~nm}$.

of $\mathrm{CS}_{1}$ by TEM and the RFC model using Raman spectroscopy results are given in Table $\mathrm{S} 1$ (see the ESI $\dagger$ ).

$\mathrm{X}$-ray diffraction (XRD) is frequently used to determine the size and structure of $\mathrm{CS}_{1} \cdot{ }^{19,20}$ Fig. 1(e) shows the background subtracted XRD signal for $\mathrm{CS}_{1}$. The broad peak may suggest a large degree of disorder in samples, but may also be due to the small size of particles. XRD of amorphous Ge (a-Ge) qdots $\left(\mathrm{FWHM}=12.68^{\circ}\right)^{27}$ are found to give a somewhat broader peak compared to the XRD here $\left(5.48^{\circ}\right.$, Fig. 1(e)). Using the Scherrer expression, ${ }^{21}$ we obtained the size of $\mathrm{CS}_{1}$ as $1.54 \mathrm{~nm}$. This is to be compared with $3.20 \mathrm{~nm}$ and $3.89 \mathrm{~nm}$ values extracted from the Raman and TEM respectively. Thus there is a clear discrepancy among TEM, the Raman and XRD results. Given that we do not observe any appreciable number of particles of sizes below $2 \mathrm{~nm}$ for $\mathrm{CS}_{1}$ in TEM (see histogram in Fig. 2(b)),

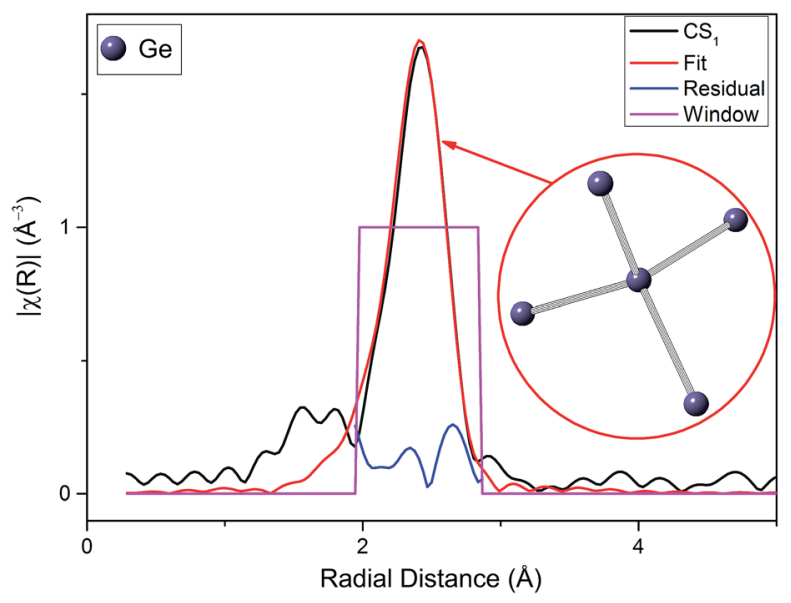

Fig. 3 FT modulus of $k^{2}$-weighted EXAFS spectrum of Ge qdots as-prepared $\left(\mathrm{CS}_{1}\right)$ at $\mathrm{Ge} \mathrm{K}$-edge shows only one peak attributed to local disorder and oxide-free surface. The figure also shows the fit, the residual between the fit and the data and the window of the fit. The models of the diamond cubic type of $\mathrm{Ge}$ is also demonstrated. The range of the models were chosen between $0-3 \AA$ for the first shells only. this suggests a degree of disorder in our samples, but not as much as in typical a-Ge. One possibility that may explain the result is a core-shell model with a small crystalline core and an
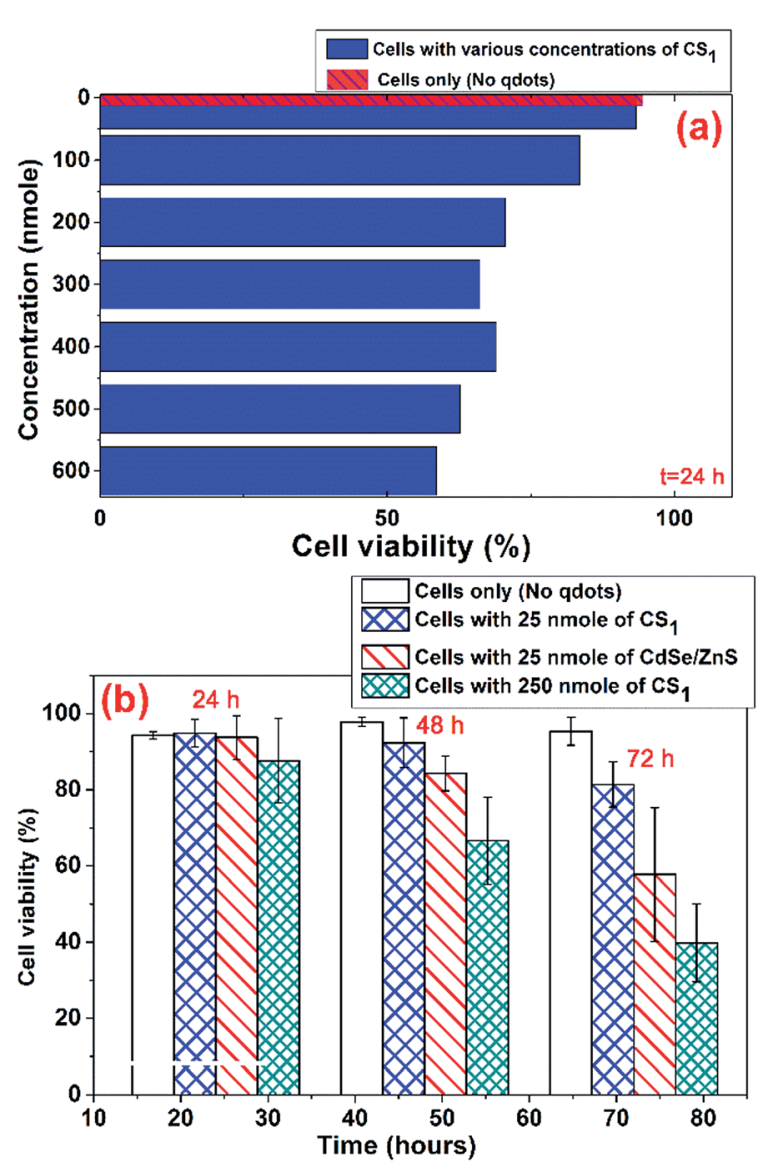

Fig. 4 Cell viability of $\mathrm{CS}_{1}$ when cultured with various concentrations of $\mathrm{CS}_{1}$ at the end of $24 \mathrm{~h}$ is shown in (a). Confirmation of the viability test of $\mathrm{CS}_{1}$ and its comparison with commercial Invitrogen (carboxyl coated $\mathrm{CdSe} / \mathrm{ZnS}$ ) qdots at 25 nmole concentration at different time points show Ge qdots are relatively promising as given in (b). 

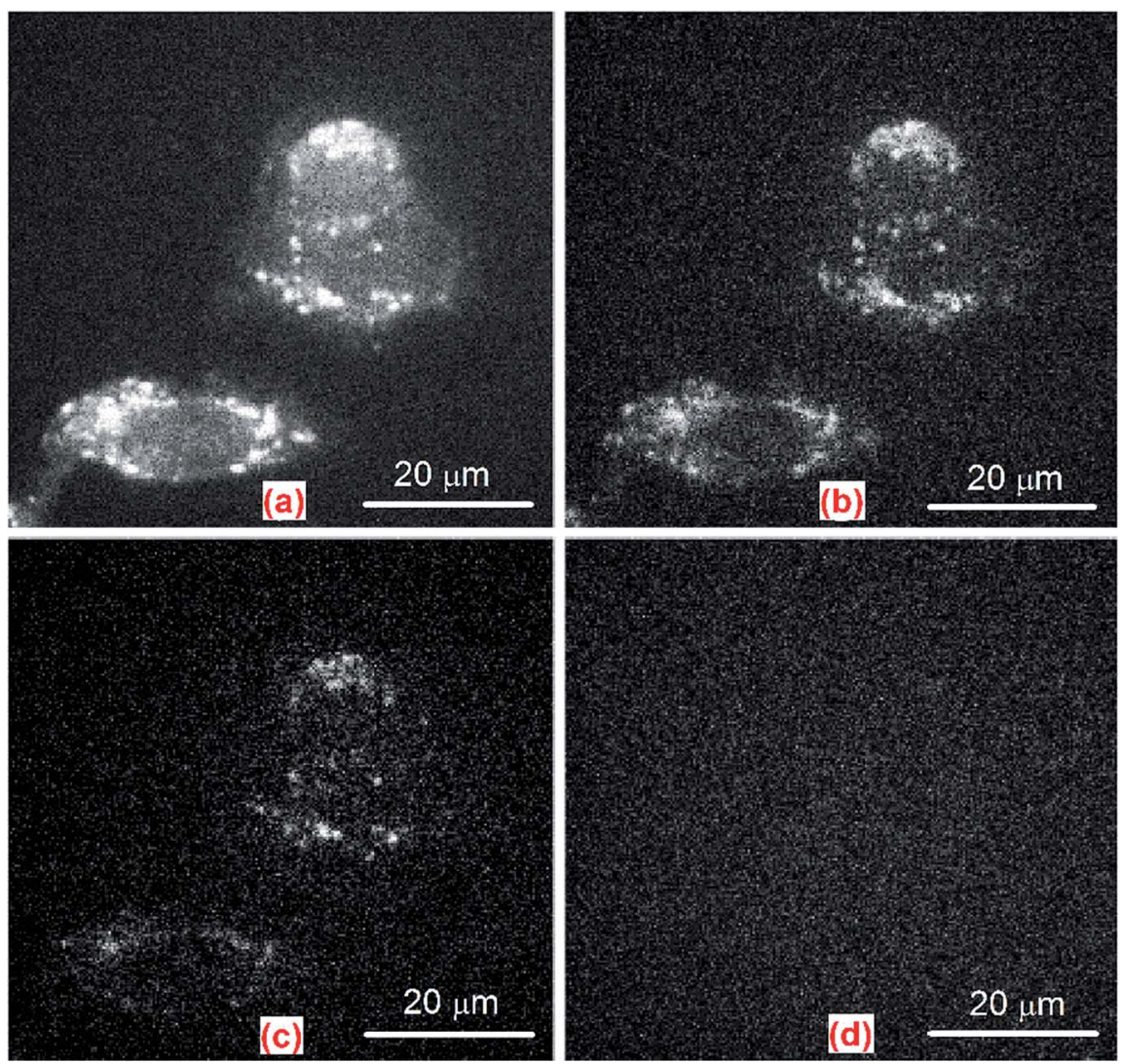

Fig. 5 Fluorescence images of $\mathrm{CS}_{1}$ in (a) to (d) acquired at various excitation wavelengths; 405, 488, 561, 647 nm shows respectively the range of optical usability. The detected laser power was around $550 \mathrm{nW}$ in the fluorescence images (see also the ESI $\uparrow$ ).

amorphous outer layer (see the inset of Fig. 1(e)). This would certainly be consistent with the theoretical modelling of small Ge qdots reported previously. ${ }^{53}$

Extended X-ray absorption fine structure (EXAFS) at Ge K-edge was used to examine the local environment around Ge atoms and shed light on the possible atomic arrangements. Fig. 3 shows the magnitude of the Fourier transform (MFT) of the $k^{2}$-weighted EXAFS signal, which provides a convenient way to visualize the average local environment around Ge. One can see that only a single peak corresponding to a Ge-Ge bond in the MFT of the EXAFS signal (the first coordination shells at $2.437 \pm 0.011 \AA$ for the diamond cubic structure - see the ESI $\dagger$ for the details of the fit) exists. The presence of only the first coordination shell in the MFT of the EXAFS signal is due to a structural disorder beyond the first coordination shell and the small size. The lack of the Ge-O related signal (at around $1.73 \AA$ ) indicates no significant amount of oxides are present in the as-prepared sample. Still, the surface must be terminated and the Ge-H symmetric stretching vibrational mode was observed (as evidence by the Raman data, see inset Fig. 1(e)). Clearly, pure H-terminated Ge qdots are unlikely to be colloidally stable due to the hydrophobic nature of the surface. Therefore, it's quite possible that colloidal stability can be achieved through the bonding of surface hydrogen to other species (e.g. ethoxide as discussed, for example, in other reports $\left.{ }^{54}\right)$.
Crystalline bulk Ge has an indirect band-gap that lies within the infrared region of energies $\left(E_{\mathrm{g}}=0.67 \mathrm{eV}, 1850 \mathrm{~nm}\right) .{ }^{55} \mathrm{In}$ order to determine the optical absorption/emission properties of any qdots, such as Ge or CdSe/ZnS, UV-Vis absorption spectroscopy and the PL spectroscopy is widely used. ${ }^{20,21,28}$ Broad UV-Vis absorption spectrum of $\mathrm{CS}_{1}$ suspended in ultra-pure water is shown in Fig. 1(f). The data show that the absorption has a shoulder at $c a .400 \mathrm{~nm}(3.1 \mathrm{eV})$. However, the PL peak is found in Fig. 1(f) at $680 \mathrm{~nm}(1.82 \mathrm{eV})$ suggesting that the nature of emission and absorption events are different. One reason for this could be due to $\mathrm{GeH}_{x} \mathrm{Cl}_{y}$ molecular clusters instead of Ge particles. This would be consistent with the Raman signatures and more importantly explain the presence of the narrow emission spectrum. Moreover, it will yield the stable emission of these molecular clusters in a cell growth environment. It is well-known that the surface contribution to the emission spectra ${ }^{19,56-58}$ may be significant due to the high probability of excitons being captured by the surface states with a subsequent recombination. On the other hand, $\mathrm{H}$-terminated a-Ge $\mathrm{Ge}^{59}(1.1 \mathrm{eV})$ was found to have larger band gaps than that of a-Ge $\mathrm{Ge}^{60}(0.5 \mathrm{eV})$ or bulk Ge $(0.67 \mathrm{ev})$. Thus, both the large amorphous intermediate region inside the qdot and the surface affect the light emission in $\mathrm{CS}_{1}$.

$\mathrm{CS}_{1}$ synthesized here represents a novel fluorescent product with a potential for bio-applications. Therefore, the biocompatibility and stability of $\mathrm{CS}_{1}$ for fluorescent imaging was tested 
on HeLa cells. First, it was necessary to understand the concentrations of Ge qdots that can be suitable for live cell applications. Cell viability as a function of Ge qdot concentration in cell growth medium is shown in Fig. 4(a). The results suggest that concentrations below 100 nmole give values of viability close to the qdot free reference. Cell number and viability were found to depend on Ge qdot concentrations and reduced relatively quickly when seeded with a higher concentration of Ge qdots of 200 nmole (see Fig. 4(a)). We further compared the viability of our un-coated as-prepared Ge qdots with commercially available (Invitrogen) carboxyl coated CdSeZnS qdots. Fig. 4(b) shows the results of the viability tests that were taken over $72 \mathrm{~h}$ and clearly indicates a higher level of biocompatibility of uncoated as-prepared Ge qdots as compared to the commercial carboxyl coated CdSe/ZnS qdots. When using smaller concentrations of the commercial carboxyl coated CdSe/ZnS qdots (e.g. 10 nmole), then the viability results show very little impact on cell viability (see Fig. S4 in ESI $\dagger$ ). There are a number of mechanisms ${ }^{61}$ reported which are related to CdSe based qdots which might affect their toxicity. Along with the leaking of Cd ions, the CdSe/ZnS qdot degradation, due to an oxide enriched environment, may result in free radicals formation which are understood to contribute to toxicity. Coating CdSe qdots with $\mathrm{ZnS}$ helps to decrease $\mathrm{Cd}$ ions desorption, although it does not stop the CdSe qdots degradation. In addition to the ZnS coating, commercial carboxyl coated CdSe/ZnS qdots might help slow the CdSe qdot degradation even further. Nevertheless, about $50 \%$ of all HeLa cells were killed after $72 \mathrm{~h}$ as shown in Fig. 4(b). However, this is not the case for Ge. It has been reported in a wide range of studies ${ }^{62-64}$ that Ge can play a therapeutic role by binding free radicals in cells via oxidation Ge.

We tested the $\mathrm{CS}_{1}$ suitability as a marker for the long term fluorescent imaging of the HeLa cells by using the spinning disk confocal microscopy. The luminescent images from the HeLa cells loaded with $\mathrm{CS}_{1}$ seem to suggest that the Ge qdots have a higher emission efficiency with the excitation wavelength of $405 \mathrm{~nm}$ rather than that of $647 \mathrm{~nm}$, at which there is nearly no emission. This result is consistent with our optical absorption measurement (Fig. 2(f)). Based on the fluorescent images in Fig. 5, it is clear that $\mathrm{CS}_{1}$ has reasonably good emission brightness when excited with an appropriate wavelength, despite having relatively lower luminescence efficiency compared to commercial carboxyl coated CdSe/ZnS qdots.

\section{Conclusions}

Reflecting upon our collected data, we were able to synthesize colloidally stable Ge qdots. Combined EXAFS, XRD, Raman and TEM measurements suggest a core-shell structure of Ge qdots with a crystalline core, an amorphous outer layer and a surface with hydrogen-related species. Using Ge qdots, the luminescent images of HeLa cells were obtained for various excitation wavelengths, which indicate that using Ge qdots as a fluorescence probe is applicable in light microscopy. We tested toxicity of Ge qdots on HeLa cells. The toxicity of Ge qdots and commercial carboxyl coated CdSe/ZnS qdots were investigated using a viability test and from this the Ge qdots were found to be the least toxic of the two. Cells with Ge qdots survived even after 3 days, which suggests that, as well as basic research, the qdots could be used in medical research, clinical imaging and drug screening trials.

\section{Acknowledgements}

William R. Little was grateful to The South East Physics Network (SEPnet). Osman Ersoy acknowledges Turkish Ministry of National Education. Helpful discussions with Prof Peter Wyatt from School of Chemistry and Biology, Queen Mary, University of London are gratefully acknowledged.

\section{References}

1 P. Alivisatos, Nat. Biotechnol., 2004, 22, 47-52.

2 T. L. Doane and C. Burda, Chem. Soc. Rev., 2012, 41, 28852911.

3 P. Mushonga, M. O. Onani, A. M. Madiehe and M. Meyer, J. Nanomater., 2012, 2012, 1-11.

4 U. Resch-genger, M. Grabolle, S. Cavaliere-jaricot, R. Nitschke and T. Nann, Nat. Methods, 2008, 5, 763-775.

5 B. J. Marcel, M. Mario, P. Gin, W. Shimon, A. A. Paul and M. Bruchez Jr, Science, 1998, 281, 2013-2016.

6 J. Fan and P. K. Chu, Small, 2010, 6, 2080-2098.

7 T. Pellegrino, S. Kudera, T. Liedl, A. Muñoz Javier, L. Manna and W. J. Parak, Small, 2005, 1, 48-63.

8 W. Chan and N. Shiao, Acta Pharmacol. Sin., 2008, 29, 259266.

9 J. Lovrić, S. J. Cho, F. M. Winnik and D. Maysinger, Chem. Biol., 2005, 12, 1227-1234.

10 G. Lin, Z. Ding, R. Hu, X. Wang, Q. Chen, X. Zhu, K. Liu, J. Liang, F. Lu, D. Lei, G. Xu and K.-T. Yong, $R S C A d v$., 2014, 4, 5792.

11 J. Liu, F. Erogbogbo, K. Yong, L. Ye, J. Liu, R. Hu and H. Chen, ACS Nano, 2013, 7(8), 7303-7310, DOI: 10.1021/ nn4029234.

12 S. Hanada, K. Fujioka, Y. Futamura, N. Manabe, A. Hoshino and K. Yamamoto, Int. J. Mol. Sci., 2013, 14, 1323-1334.

13 L. T. Canham, Appl. Phys. Lett., 1990, 57, 1046.

14 A. G. Cullis and L. T. Canham, Nature, 1991, 353, 335-338.

15 Y. Maeda, N. Tsukamoto, Y. Yazawa, Y. Kanemitsu and Y. Masumoto, Appl. Phys. Lett., 1991, 59, 3168.

16 A. P. Alivisatos, Science, 1996, 271, 933-937.

17 H. Yang, X. Wang, H. Shi, F. Wang, X. Gu and X. Yao, J. Cryst. Growth, 2002, 236, 371-375.

18 J. M. Buriak, Chem. Rev., 2002, 102, 1271-1308.

19 C. Delerue, G. Allan and M. Lannoo, J. Lumin., 1998, 80, 6573.

20 J. R. Heath, J. J. Shiang and A. P. Alivisatos, J. Chem. Phys., 1994, 101, 1607-1615.

21 B. R. Taylor, S. M. Kauzlarich, G. R. Delgado and H. W. H. Lee, Chem. Mater., 1999, 11, 2493-2500.

22 B. R. Taylor, G. A. Fox, L. J. Hope-Weeks, R. S. Maxwell, S. M. Kauzlarich and H. W. Lee, Mater. Sci. Eng., B, 2002, 96, 90-93. 
23 V. Karavanskii, A. Lomov, A. Sutyrin, V. Bushuev, N. Loikho, N. Melnik, T. Zavaritskaya and S. Bayliss, Thin Solid Films, 2003, 437, 290-296.

24 N. Myung, X. Lu, K. P. Johnston and A. J. Bard, Nano Lett., 2004, 4, 183-185.

25 X. Lu, B. A. Korgel and K. P. Johnston, Nanotechnology, 2005, 16, S389-S394.

26 E. J. Henderson, C. M. Hessel and J. G. C. Veinot, J. Am. Chem. Soc., 2008, 130, 3624-3632.

27 N. H. Chou, K. D. Oyler, N. E. Motl and R. E. Schaak, Chem. Mater., 2009, 21, 4105-4107.

28 D. D. Vaughn, J. F. Bondi and R. E. Schaak, Chem. Mater., 2010, 22, 6103-6108.

29 D. A. Ruddy, J. C. Johnson, E. R. Smith and N. R. Neale, ACS Nano, 2010, 4, 7459-7466.

30 E. Muthuswamy, A. S. Iskandar, M. M. Amador and S. M. Kauzlarich, Chem. Mater., 2013, 25, 1416-1422.

31 R. Hardman, Environ. Health Perspect., 2006, 114, 165-172.

32 S. Sato, S. Nozaki, H. Morisaki and M. Iwase, Appl. Phys. Lett., 1995, 66, 3176.

33 S. Nozaki, S. Sato, S. Rath, H. Ono and H. Morisaki, Bull. Mater. Sci., 1999, 22, 377-381.

34 S. B. Qadri, E. F. Skelton and A. W. Webb, J. Appl. Phys., 1983, 54, 3609.

35 Y. J. Cho, H. S. Im, H. S. Kim, Y. Myung, S. H. Back, Y. R. Lim, C. S. Jung, D. M. Jang, J. Park, E. H. Cha, W. Il Cho, F. Shojaei and H. S. Kang, ACS Nano, 2013, 7, 9075-9084.

36 J. Liu, C. Liang, Z. Tian, S. Zhang and G. Shao, Sci. Rep., 2013, 3, 1741.

37 J. Joannopoulos and M. Cohen, Phys. Rev. B: Solid State, 1973, 7, 2644-2657.

38 T. N. Lambert, N. L. Andrews, H. Gerung, T. J. Boyle, J. M. Oliver, B. S. Wilson and S. M. Han, Small, 2007, 3, 691-699.

39 S. Prabakar, A. Shiohara, S. Hanada, K. Fujioka, K. Yamamoto and R. D. Tilley, Chem. Mater., 2010, 22, 482-486.

40 A. J. Dent, G. Cibin, S. Ramos, A. D. Smith, S. M. Scott, L. Varandas, M. R. Pearson, N. A. Krumpa, C. P. Jones and P. E. Robbins, J. Phys.: Conf. Ser., 2009, 190, 012039.

41 B. Ravel and M. Newville, J. Synchrotron Radiat., 2005, 12, 537-541.

42 S. Zabinsky, J. Rehr, A. Ankudinov, R. Albers and M. Eller, Phys. Rev. B: Condens. Matter Mater. Phys., 1995, 52, 29953009.
43 C. E. Bottani, C. Mantini, P. Milani, M. Manfredini, A. Stella, P. Tognini, P. Cheyssac and R. Kofman, Appl. Phys. Lett., 1996, 69, 2409.

44 M. Fujii, S. Hayashi and K. Yamamoto, Jpn. J. Appl. Phys., 1991, 30, 687-694.

45 I. H. Campbell and P. M. Fauchet, Solid State Commun., 1986, 58, 739-741.

46 H. Richter, Z. P. Wang and L. Ley, Solid State Commun., 1981, 39, 625-629.

47 V. Paillard, P. Puech, M. A. Laguna, R. Carles, B. Kohn and F. Huisken, J. Appl. Phys., 1999, 86, 1921.

48 Y. Sasaki and C. Horie, Phys. Rev. B: Condens. Matter Mater. Phys., 1993, 47, 3811-3818.

49 R. Kobliska, S. Solin, M. Selders, R. Chang, R. Alben, M. Thorpe and D. Weaire, Phys. Rev. Lett., 1972, 29, 725-728. 50 C. Su, C.-S. Tsai, C.-E. Lin, K.-H. Chen, J.-K. Wang and J.-C. Lin, Surf. Sci., 2000, 445, 139-150.

51 C. Mui, J. H. Han, G. T. Wang, C. B. Musgrave and S. F. Bent, J. Am. Chem. Soc., 2002, 124, 4027-4038.

52 F. Maroun, F. Ozanam and J.-N. Chazalviel, J. Phys. Chem. B, 1999, 103, 5280-5288.

53 L. Pizzagalli, G. Galli, J. Klepeis and F. Gygi, Phys. Rev. B: Condens. Matter Mater. Phys., 2001, 63, 165324.

54 E. J. Henderson, M. Seino, D. P. Puzzo and G. A. Ozin, ACS Nano, 2010, 4, 7683-7691.

55 E. Bianco, S. Butler, S. Jiang, O. D. Restrepo, W. Windl and J. E. Goldberger, ACS Nano, 2013, 7, 4414-4421.

56 A. P. Alivisatos, J. Phys. Chem., 1996, 100, 13226-13239.

57 S. Okamoto and Y. Kanemitsu, Phys. Rev. B: Condens. Matter Mater. Phys., 1996, 54, 16421-16424.

58 J. H. Warner and R. D. Tilley, Nanotechnology, 2006, 17, 3745-3749.

59 I. Chambouleyron, C. F. Graeff, A. R. Zanatta, F. Fajardo, M. Mulato, R. Campomanes, D. Comedi and F. C. Marques, Phys. status solidi, 1995, 192, 241-251.

60 T. Donovan, W. Spicer and J. Bennett, Phys. Rev. Lett., 1969, 22, 1058-1061.

61 T. Jamieson, R. Bakhshi, D. Petrova, R. Pocock, M. Imani and A. M. Seifalian, Biomaterials, 2007, 28, 4717-4732.

62 S. Goodman, Med. Hypotheses, 1988, 26, 207-215.

63 A. Fujii, N. Kuboyama, J. Yamane, S. Nakao and Y. Furukawa, Gen. Pharmacol., 1993, 24, 1527-1532.

64 B. Kamen, A New Approach to Immunity, Nutrition Encounter, 8th edn, October 1987, vol. 184.

65 F. Coppari, A. Di Cicco, A. Congeduti, J. C. Chervin, F. Baudelet and A. Polian, High Pressure Res., 2009, 29, 103-107. 